\title{
Nouveaux cas de ségrégation indépendante de caractères mendéliens chez le pêcher
}

René MONET \& Yves BASTARD

I.N.R.A., Station d'Arboriculture fruitière, Centre de Recherches de Bordeaux F 33140 Pont-de-la-Maye.

RÉSUMÉ

Cette étude fait état des observations effectuées dans les générations $F_{1}$ et $F_{2}$ issues de deux croisements : « Redhaven » $x$ "Silver Lode » et « J.H. Hale » $x$ " Amsden ».

Elle montre la ségrégation indépendante de 3 couples de gènes déterminant la forme des fleurs (Sh sh), la pilosité de l'épiderme du fruit ( $\mathrm{G} \mathrm{g})$, la couleur de sa chair ( $\mathrm{Y}$ y) d'une part et de 3 couples de gènes déterminant la forme des nectaires foliaires (E e), la stérilité mâle (Ps ps) et la couleur de la chair (Y y) d'autre part.

Mots-clés additionnels : Hérédité, Caractères simples, Fleur, Fruit, Nectaires foliaires, Stérilité mâle.

New cases of independent segregation for Mendelian characters in peach.

The observations in this study deal with the $\mathrm{F}_{1}$ and $\mathrm{F}_{2}$ generations obtained by crossing the cultivars : « Redhaven » $x$ 《Silver Lode » and « J.H. Hale » $x$ « Amsden ".

It shows the independent segregation of 3 pairs of genes which determine flower form (Sh sh), smooth-skinned fruit $(\mathrm{G} \mathrm{g})$, and flesh color ( $\mathrm{Y} y)$ on the one hand 3 pairs of genes which determine the form of foliar glands ( $\mathrm{E}$ e), pollen sterility (Ps ps) and flesh color ( $\mathrm{Y}$ y).

Additional key-words : Heredity, Flower, Fruit, Foliar glands, Male sterility.

\section{INTRODUCTION}

Chez le pêcher, on peut faire état de 22 caractères à hérédité simple, monogénique ou bigénique. Cependant, l'étude des liaisons pouvant exister entre les locus portant ces gènes n'en est qu'à ses débuts : 8 groupes de linkage peuvent exister théoriquement chez cette espèce dont le nombre chromosomique est $2 n=16$. BAILEY \& FRENCH (1949) ont proposé un premier groupe de linkage qui n'est probablement pas correct (MONET, 1965). CRANE \& LAWRENCE (1956) ont donné à titre d'exemple le tableau de contingence d'une $\mathrm{F}_{2}$ concernant la forme des nectaires foliaires et la pilosité de l'épiderme dans le cas où ces caractères auraient une disjonction indépendante mais ces auteurs ne semblent pas avoir observé cette disjonction. Enfin nous-mêmes avons décrit (MONET, 1967 ; MONET \& BASTARD, 1972) plusieurs cas de disjonctions indépendantes.

Nous présentons ici une nouvelle contribution à cet aspect de la génétique du pêcher.

\section{MATÉRIEL ET MÉTHODES}

Notre étude a pour point de départ 2 croisements. Le $1^{\mathrm{er}}$, réalisé en 1969, « Redhaven 》 X «Silver Lode », a donné 46 arbres ; le $2^{\mathrm{e}}$, réalisé en 1970 , « J.H. Hale » $\times$ « Ams- den ", a donné 233 arbres. Chaque arbre de ces familles $F_{1}$ a été numéroté séparément.

Pour constituer les générations $F_{2}$, nous avons autofécondé les arbres 9, 20 et 46 du croisement « Redhaven » $x$ « Silver Lode » et les arbres 94, 102, 111 et 210 du croisement « J.H. Hale » $x$ « Amsden ». Ce choix tient à leur valeur agronomique mais aussi, dans le cas du $2^{e}$ croisement, à des considérations génétiques qui seront justifiées par la suite.

Le croisement « Redhaven " $x$ 《 Silver Lode " permettait l'observation en $F_{2}$ des disjonctions dans lesquelles étaient impliqués les caractères mendéliens suivants :

- forme de la fleur : il existe chez le pêcher des fleurs campanulées (génotypes Sh Sh* ou Sh sh) et des fleurs rosacées (génotypes sh sh) (BAILEY \& FRENCH, 1942).

- présence ou absence de poils sur l'épiderme du fruit : le caractère fruit à épiderme duveteux est dominant (génotypes $\mathrm{GG}$ ou $\mathrm{Gg}$ ) ; le caractère fruit à épiderme glabre (nectarines et brugnons) est récessif (génotype gg) (BLAKE, 1933).

- couleur de la chair : le caractère chair blanche est dominant (génotypes YY ou Yy), le caractère chair jaune est récessif (génotype yy) (CONNORS, 1920).

* Les symboles que nous utilisons sont ceux donnés par le premier auteur qui a étudié le caractère ou, à défaut, ceux proposés par BAILEY \& FRENCH (1949); ils correspondent aux initiales de : Showy (Sh, $\mathrm{sh})$, Glabrous $(\mathrm{G}, \mathrm{g})$, Yellow $(\mathrm{Y}, \mathrm{y})$, Eglandular (E, e), Pollen sterility (Ps, ps). 
Nous avions autofécondé « Redhaven » en 1967. La famille d'autofécondation a permis de vérifier que ce géniteur à fleurs campanulées, dont le fruit a un épiderme duveteux et une chair jaune, est homozygote pour ces 3 caractères. Le génotype de " Redhaven » est donc: Sh Sh, GG, yy.

De la même manière, l'autofécondation de "Silver Lode » réalisée en 1969 et qui avait donné 13 arbres, a permis de vérifier que ce géniteur à fleurs rosacées, à épiderme glabre (nectarine) et à chair blanche est homozygote pour ces caractères. Le génotype de "Silver Lode " est donc: sh sh, gg, Y Y.

Dans le croisement « J.H. Hale » $X$ « Amsden », nous nous sommes intéressés aux caractères suivants :

- nectaires foliaires : il existe pour ce caractère 3 possibilités : les nectaires peuvent être réniformes (génotype $E \mathrm{E}$ ), circulaires (génotype $E$ e) ou absents (génotype e e) ; c'est un cas de dominance incomplète (CONNORS, 1922).

- stérilité mâle : le caractère mâle fertile est dominant (génotypes Ps Ps ou Ps ps); le caractère mâle stérile est récessif (génotypes ps ps) (SCOTT \& WEINBERGER, 1944).

- couleur de la chair du fruit : nous avons donné plus haut les situations génotypiques possibles pour ce caractère.

« J.H. Hale » a des nectaires réniformes, est mâle stérile et produit des fruits à chair jaune. Son génotype est donc : EE psps yy. « Amsden » a des nectaires circulaires, est mâle fertile et a des fruits à chair blanche, son génotype peut s'écrire : Ee PsPs YY, Ee PsPs Yy, Ee Psps Yy ou Ee Psps YY.

L'incertitude qui pèse sur le génotype de "Amsden " pour les caractères de stérilité mâle et de couleur de la chair du fruit pourra être levée à la $F_{1}$ puisque le parent « J.H. Hale » est homozygote récessif pour ces 2 caractères.

\section{RÉSULTATS}

\section{A. Caractéristiques des hybrides $\mathbf{F}_{1}$}

\section{1. $F_{1}$ « Redhaven » $\times$ « Silver Lode »}

La famille est uniforme pour les caractères étudiés. Les 46 arbres ont tous des fleurs campanulées et donnent des pêches à chair blanche ; leur génotype est donc : Shsh Gg Yy.

\section{2. $F_{1} « J . H$. Hale $» \times$ « Amsden »}

La famille est homogène pour le caractère mâle fertile et pour la couleur blanche de la chair, par contre, la moitié des arbres environ ( 81 sur 169 observés) est à nectaires réniformes et l'autre moitié à nectaires circulaires.

Les génotypes des arbres de cette $F_{1}$ sont donc de 2 sortes :

EE Psps Yy pour les arbres à nectaires réniformes

Ee Psps Yy pour les arbres à nectaires circulaires.

L'uniformité de la $F_{1}$ pour les caractères mâle fertile et chair blanche prouve que le parent «Amsden » était homozygote pour ces caractères. Sa formule génotypique est donc Ee, PsPs, YY.

Pour réaliser la $F_{2}$, nous avons choisi d'autoféconder les arbres 94, 102, 111 et 210 dont les nectaires étaient circulaires, leur formule génétique était donc : Ee, Psps, Yy ce qui nous permettait l'étude des liaisons entre ces 3 couples d'allèles.

\section{B. Etude des disjonctions observées dans la $F_{2}$}

1. $F_{2}$ «Redhaven » $\times$ «Silver Lode »

Le tableau 1 donne la ségrégation phénotypique obtenue pour les caractères : forme de la fleur, pilosité du fruit, couleur de la chair du fruit. Bien que la dimension de la famille soit modeste, les chiffres observés sont statistiquement assimilables à ceux d'une disjonction de 3 paires de caractères allélomorphes indépendants.

\section{2. $F_{2} \ll J . H$. Hale $» \times$ «Amsden 》}

Le tableau 2 donne la ségrégation phénotypique obtenue pour les caractères : forme des nectaires foliaires, stérilité mâle, couleur de la chair du fruit. Là encore, les chiffres observés sont statistiquement assimilables à une disjonction de 3 paires de caractères allélomorphes indépendants.

\section{CONCLUSION}

L'étude des disjonctions dans les deux familles $\mathrm{F}_{2}$ montre que les 3 couples de gènes Sh sh, Gg, Yy d'une part et

TABLEAU 1

Répartition des phénotypes dans la famille $F_{2}$ issue du croisement « Redhaven » $\times$ "Silver Lode » Phenotypic distribution in the $F_{2}$ family from the cross " Redhaven » $\times$ « Silver Lode »

\begin{tabular}{|c|c|c|c|c|c|c|c|c|}
\hline & \multicolumn{4}{|c|}{ Fleur campanulée } & \multicolumn{4}{|c|}{ Fleur rosacée } \\
\hline \multirow{2}{*}{ Fréquences } & \multicolumn{2}{|c|}{$\begin{array}{c}\text { Fruit à épiderme } \\
\text { duveteux }\end{array}$} & \multicolumn{2}{|c|}{$\begin{array}{l}\text { Fruit à épiderme } \\
\text { glabre }\end{array}$} & \multicolumn{2}{|c|}{$\begin{array}{l}\text { Fruit à épiderme } \\
\text { duveteux }\end{array}$} & \multicolumn{2}{|c|}{$\begin{array}{l}\text { Fruit à épiderme } \\
\text { glabre }\end{array}$} \\
\hline & $\begin{array}{l}\text { Chair } \\
\text { blanche }\end{array}$ & $\begin{array}{l}\text { Chair } \\
\text { jaune }\end{array}$ & $\begin{array}{l}\text { Chair } \\
\text { blanche }\end{array}$ & $\begin{array}{l}\text { Chair } \\
\text { jaune }\end{array}$ & $\begin{array}{l}\text { Chair } \\
\text { blanche }\end{array}$ & $\begin{array}{l}\text { Chair } \\
\text { jaune }\end{array}$ & $\begin{array}{l}\text { Chair } \\
\text { blanche }\end{array}$ & $\begin{array}{l}\text { Chair } \\
\text { jaune }\end{array}$ \\
\hline observées & 165 & 46 & 35 & 19 & 58 & 16 & 26 & 8 \\
\hline $\begin{array}{l}\text { théoriques } \\
\text { (disjonction : } \\
\text { 27.9.9.9.3.3.3.1) }\end{array}$ & 156,6 & 52,2 & 52,2 & 17,4 & 52,2 & 17,4 & 17,4 & 5,8 \\
\hline
\end{tabular}


TABLEAU 2

Répartition des phénotypes dans la famille $F_{2}$ issue du croisement "J.H. Hale " $\times$ "Amsden " Phenotypic distribution in the $F_{2}$ family from the cross "J.H. Hale " $\times$ « Amsden "

\begin{tabular}{|c|c|c|c|c|c|c|c|c|c|c|c|c|}
\hline \multirow[b]{3}{*}{ Fréquences } & \multicolumn{4}{|c|}{ Nectaires réniformes } & \multicolumn{4}{|c|}{ Nectaires circulaires } & \multicolumn{4}{|c|}{ Nectaires absents } \\
\hline & \multicolumn{2}{|c|}{ Mâle fertile } & \multicolumn{2}{|c|}{ Mâle stérile } & \multicolumn{2}{|c|}{ Mâle fertile } & \multicolumn{2}{|c|}{ Mâle stérile } & \multicolumn{2}{|c|}{ Mâle fertile } & \multicolumn{2}{|c|}{ Mâle stérile } \\
\hline & $\begin{array}{l}\text { Chair } \\
\text { blanche }\end{array}$ & $\begin{array}{l}\text { Chair } \\
\text { jaune }\end{array}$ & $\begin{array}{l}\text { Chair } \\
\text { blanche }\end{array}$ & $\begin{array}{l}\text { Chair } \\
\text { jaune }\end{array}$ & $\begin{array}{l}\text { Chair } \\
\text { blanche }\end{array}$ & $\begin{array}{l}\text { Chair } \\
\text { jaune }\end{array}$ & $\begin{array}{l}\text { Chair } \\
\text { blanche }\end{array}$ & $\begin{array}{l}\text { Chair } \\
\text { jaune }\end{array}$ & $\begin{array}{l}\text { Chair } \\
\text { blanche }\end{array}$ & $\begin{array}{l}\text { Chair } \\
\text { jaune }\end{array}$ & $\begin{array}{l}\text { Chair } \\
\text { blanche }\end{array}$ & $\begin{array}{l}\text { Chair } \\
\text { jaune }\end{array}$ \\
\hline observées & 27 & 10 & 14 & 3 & 69 & 16 & 18 & 5 & 33 & 16 & 9 & 1 \\
\hline $\begin{array}{l}\text { théoriques } \\
\text { (disjonction } \\
\text { 18.9.9.6.6.3.3.3.3.2.1.1.) }\end{array}$ & 31,05 & 10,35 & 10,35 & 3,45 & 62,1 & 20,7 & 20,7 & 6,8 & 31,05 & 10,35 & 10,35 & 3,45 \\
\hline
\end{tabular}

$X^{2}$ calculé $=10,05$ non significatif au seuil 5 p. $100\left(X^{2} 0,05=19,68\right.$ pour 11 degrés de liberté).

\section{TABLEAU 3}

Cas de ségrégations indépendantes des caractères mendéliens du pêcher

Cases of independent segregation in peach for mendelian characters

\begin{tabular}{|c|c|c|c|c|c|c|c|}
\hline & $\begin{array}{c}\text { Anthocyanes } \\
\text { normal/ } \\
\text { déficient }\end{array}$ & $\begin{array}{l}\text { Nectaires } \\
\text { foliaires/ } \\
\text { réniformes } \\
\text { circulaires/ } \\
\text { absents }\end{array}$ & $\begin{array}{c}\text { Fleur } \\
\text { campanulée/ } \\
\text { rosacée }\end{array}$ & $\begin{array}{l}\text { Mâle } \\
\text { fertile/ } \\
\text { stérile }\end{array}$ & $\begin{array}{l}\text { Epiderme } \\
\text { duveteux/ } \\
\text { glabre }\end{array}$ & $\begin{array}{c}\text { Chair } \\
\text { blanche/ } \\
\text { jaune }\end{array}$ & $\begin{array}{c}\text { Non pavie } \\
\text { pavie }\end{array}$ \\
\hline \multicolumn{8}{|l|}{$\begin{array}{l}\text { Anthocyanes } \\
\text { normal/déficient }\end{array}$} \\
\hline $\begin{array}{l}\text { Nectaires } \\
\text { foliaires/réniformes/ } \\
\text { circulaires/absents }\end{array}$ & $?$ & & & & & & \\
\hline $\begin{array}{l}\text { Fleur } \\
\text { campanulée/rosacée }\end{array}$ & $\begin{array}{c}\text { Disjonction } \\
\text { indépendante }\end{array}$ & $\begin{array}{c}\text { Disjonction } \\
\text { indépendante }\end{array}$ & & & & & \\
\hline $\begin{array}{l}\text { Mâle } \\
\text { fertile/stérile }\end{array}$ & $?$ & $\begin{array}{c}\text { Disjonction } \\
\text { indépendante }\end{array}$ & $?$ & & & & \\
\hline $\begin{array}{l}\text { Epiderme } \\
\text { duveteux/glabre }\end{array}$ & $?$ & $?$ & $\begin{array}{l}\text { Disjonction } \\
\text { indépendante }\end{array}$ & $?$ & & & \\
\hline $\begin{array}{l}\text { Chair } \\
\text { blanche/jaune }\end{array}$ & $?$ & $\begin{array}{c}\text { Disjonction } \\
\text { indépendante }\end{array}$ & $\begin{array}{c}\text { Disjonction } \\
\text { indépendante }\end{array}$ & $\begin{array}{c}\text { Disjonction } \\
\text { indépendante }\end{array}$ & $\begin{array}{l}\text { Disjonction } \\
\text { indépendante }\end{array}$ & & \\
\hline $\begin{array}{l}\text { Non pavie/ } \\
\text { pavie }\end{array}$ & $\begin{array}{l}\text { Disjonction } \\
\text { indépendante }\end{array}$ & $?$ & $\begin{array}{l}\text { Disjonction } \\
\text { indépendante }\end{array}$ & $?$ & $?$ & $?$ & \\
\hline
\end{tabular}

les 3 couples de gènes Ee, Ps ps, Yy, d'autre part, se disjoignent comme des couples indépendants ce qui signifie que les locus qui portent ces gènes sont sur des chromosomes différents ou sur le même chromosome à plus de 50 unités de crossing over. Nous avons déjà montré que les associations Sh, sh, Yy et Ee, Ps ps se disjoignent indépendamment (MONET, 1967 ; MONET \& BASTARD, 1972). Cette étude confirme donc ces résultats et en apporte 4 nouveaux concernant les associations : Sh sh et $\mathrm{Gg}, \mathrm{Gg}$ et $\mathrm{Yy}, \mathrm{Ee}$ et
Yy, Ps ps et Yy. Le tableau 3 fait état de l'ensemble des associations que nous avons étudiées. Nous n'avons pas jusqu'ici trouvé de cas de linkage mais il reste encore de nombreuses combinaisons à étudier.

Si l'intérêt de ces résultats reste limité pour le sélectionneur, ils contribuent cependant à élargir la connaissance génétique de l'espèce.

Reçu le 7 octobre 1982. Accepté le 22 novembre 1982. 


\section{RÉFÉRENCES BIBLIOGRAPHIQUES}

Bailey J.S., French A.P., 1942. The inheritance of blossom type and blossom size in the peach. Proc. Am. Soc. Hort. Sci., 40, 248-250.

Bailey J.S., French A.P, 1949. The inheritance of certain fruit and foliage characters in the peach. Mass. Agr. Exp. Sta. Bull., 452, 1-31.

Blake M.A., 1933, The "J.H. Hale " as a parent in peach crosses. Proc. Am. Soc. Hort. Sci., 29, 131-136.

Connors C.H., 1920. Some notes on the inheritance of unit characters in the peach. Proc. Am. Soc. Hort. Sci., 16, 24-36.

Connors C.H., 1922. Inheritance of foliar glands of the peach. Proc. Am. Soc. Hort. Sci., 18, 20-26.
Crane M.B., Lawrence W.J.C., 1938. The genetics of garden plants 7-9. Mac Millan and Co Limited, London, 287 p.

Monet R., 1965. Caractères à déterminisme génétique simple chez Prunus persica. Ann. Amélior. Plantes, 15, 99-106.

Monet R., 1967. Contribution à l'étude génétique du pêcher. Ann. Amélior. Plantes, 17, 5-11.

Monet R., Bastard Y., 1972. Contribution à l'étude du contrôle génétique de quelques caractères morphologiques chez le pêcher. Ann. Amélior. Plantes, 22, 399-483.

Scott D.H., Weinberger J.H., 1944. Inheritance of pollen sterility some peach varieties. Proc. Am. Soc. Hort. Sci, 45, 229-232. 